\title{
Rendimientos económicos de las mujeres mexicanas jefas de familia, como asalariadas y empresarias
}

\author{
Mexican breadwinner women's economic returns, \\ such as working women or entrepreneurs
}

Pablo Daniel Palacios Duarte ${ }^{1}$, María Luisa Saavedra García ${ }^{2}$ y Teresa de Jesús Vargas Vega

Fecha de recepción: 19 de agosto de 2020

Fecha de aceptación: 25 de noviembre de 2020

1- Nacionalidad: Mexicana. Grado: Doctorado en Economía de la Empresa. Adscripción: Benemérita Universidad Autónoma de Puebla. (DORCID: https://orcid.org/0000-0003-3741-770X. Correo electrónico: palaciosduarte@hotmail.com

2- Nacionalidad: Mexicana. Grado: Doctorado en Administración. Adscripción: Universidad Nacional Autónoma de México. (DORCID: https://orcid.org/0000-0002-3297-1157. Correo electrónico: maluisasaavedra@yahoo.com

3- Nacionalidad: Mexicana. Grado: Doctorado en Economía. Adscripción: Universidad Autónoma del Estado de Hidalgo. (DORCID: http://orcid.org/0000-0002-6051-7197. Correo electrónico: tvargasv@gmail.com 


\section{Resumen}

El objetivo de esta investigación consistió en comparar el rendimiento de las actividades económicas de las mujeres mexicanas jefas de familia, para determinar si existen diferencias en los rendimientos de la fuente de trabajo ya sea subordinado (asalariada) o independiente (empresaria), así como saber qué las impulsa a emprender. Se trabajó con una muestra de 7,411 mujeres asalariadas y 1,688 independientes (empresarias), y se empleó un modelo de dos etapas (Probit y MCO). Nuestros hallazgos muestran que sí existen diferencias en el rendimiento de las mujeres asalariadas y empresarias, siendo menores los de estas últimas. Conjuntamente, se encontró que equilibrar el cuidado de los hijos menores, y el bajo ingreso que percibe siendo asalariada como consecuencia del bajo nivel educativo que posee; serían las principales motivaciones de la mujer para ser empresaria, sin embargo, su prioridad no es la riqueza económica.

Palabras clave: emprendimiento, utilidades, género, techo de cemento.

\section{Abstract}

The objective of this research was to compare the Mexican breadwinner women's economic activities performance, to determine if there are differences in the returns depending on the source of work, whether subordinate (working woman) or independent (businesswoman), furthermore to know what encourages women to be entrepreneurs. We worked with a sample with 7,411 working women and 1,688 businesswomen and used a two-stage model (Probit and MCO). Our findings revealed differences between economic returns, depending on the source of work, businesswomen had the lowest returns. Balance of childcare and low incomes that women earn as a working person, caused by the low educational level, it is the principal motivation for women to be businesswomen. However, their priority is not economic wealth.

Keywords: entrepreneurship, utilities, gender, cement roof. 


\section{Introducción}

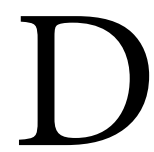

e acuerdo con la Encuesta Nacional de Ingresos y Gastos de los Hogares (INEGI, 2017), en Mé-

xico uno de cada cuatro hogares mexicanos tiene como jefe de familia a una mujer, liderando

esta estadística el estado de Sonora y la Ciudad de México con 36\% y 35\%, respectivamente. Según los estudiosos del tema, este tipo de hogares van en aumento en México y están caracterizados por su estado de pobreza y vulnerabilidad (Altamirano, Morayta y Mabire, 2020; Farias et al., 2014; García y De Oliveira, 2005); entre las razones para el aumento de estos hogares se encuentran el incremento en la esperanza de vida de las mujeres; la acentuación de las separaciones, divorcios, abandonos por parte de la pareja masculina, migración, los embarazos adolescentes donde los hombres no asumen su responsabilidad, esto como consecuencia de fenómenos económicos y culturales donde se destaca el fuerte vínculo de la madre con los hijos (Farias et al., 2014; García y De Oliveira, 2005). Por otra parte, se encuentra el incremento de la escolaridad (Lechuga et al., 2018), el control natal (González et al., 2020), y la participación laboral de las mujeres que hacen más viable la ruptura cuando se encuentran en una relación poco satisfactoria o violenta, asumiendo así la jefatura del hogar (Veloz, 2017).

De este modo, las jefas de familia se convierten en las proveedoras del hogar, lo cual implica contar con un trabajo remunerado o actividad económica independiente que les permita recibir los ingresos suficientes para cubrir los gastos familiares (Farias et al., 2014; Jiménez y Hernández, 2020; Veloz, 2017); sin que esto las exima de sus responsabilidades de cuidado, educación de los hijos y trabajo doméstico, por lo que trabajan doble jornada por la multiplicidad de funciones que realizan (Lázaro et al., 2007). Es aquí donde surge la interrogante de si es más rentable para estas jefas de familia tener un trabajo dependiente, donde pueden recibir un salario o dedicarse a una actividad económica independiente como empresarias, y lo que impulsa a estas últimas a emprender. Lo anterior, dado que la Agenda 2030 de Desarrollo Sostenible (2020) hace patente la necesidad de impulsar el crecimiento económico con base en el pleno empleo productivo, el trabajo decente y la equidad de género.

Así pues, el objetivo de esta investigación consistió en comparar el rendimiento de los ingresos de las mujeres asalariadas y de aquellas que trabajan de forma independiente, bajo el supuesto de que los determinantes de elección son la búsqueda de la riqueza y/o la necesidad de equilibrar sus actividades económicas con sus responsabilidades familiares, con el propósito de conocer los efectos que poseen estos elementos en los rendimientos obtenidos por mujeres.

\section{Revisión de la Literatura}

\subsection{Mujeres jefas de hogar}

De las investigaciones realizadas en México, García y Oliveira (2005) encontró que las jefas de la familia realizan labores domésticas del mismo modo o incluso más ampliamente que las mujeres que las que no lo son; además, asumen la responsabilidad de la organización de la vida familiar, realizan labores de cuidado y trámites; son las que toman las decisiones en su familia; lamentablemente, una 
alta proporción estuvieron expuestas a una situación de violencia doméstica por parte de sus parejas (Sánchez y Ravelo, 2013; Veloz, 2017).

Lázaro et al. (2007) observaron que, para las mujeres, el trabajo extra doméstico les brinda grandes satisfacciones, por los logros de su esfuerzo individual y trabajo; por sus múltiples funciones buscan establecer una red de apoyo familiar, con madres, abuelas, tías, lo que aminora un poco la tensión al ausentarse del espacio familiar. También, refieren que, aunque al inicio se insertan al mercado laboral por necesidades de diversa índole, después cambia su orientación de desarrollo profesional por un mejor salario, un mejor puesto, buscando elevar su calidad de vida y la de sus dependientes, accediendo así a espacios de poder de donde han sido históricamente excluidas (Sharma, 2013; Sharma y Varma, 2008).

En otro estudio realizado por Farias et al. (2014) hallaron que las jefas de familia en la Ciudad de México tenían en promedio 33.5 años, poseían estudios de nivel medio superior o superior más de la mitad de ellas, el 69.5\% desempeñaban una actividad remunerada ya sea dependiente o independiente, el 56.7\% estaban casadas o en unión libre, y el 74.1\% tenían hijos.

Escamilla et al. (2013), en su investigación realizada en Chile, encontraron que las jefas de hogar buscan como meta alcanzar una estabilidad tanto económica como laboral, con un horario flexible para alcanzar un buen desempeño en el trabajo, sin descuidar a sus hijos; así también, el independizarse a través de un emprendimiento, para compatibilizar mejor el trabajo con el cuidado de los hijos.

\subsection{Emprendimiento femenino, razones para emprender}

Guzmán y Rodríguez (2008), el Banco Internacional de Reconstrucción (BIRF, 2010), la Organización Internacional de Trabajo (OIT, 2015), Machado et al. (2016), Mejía et al. (2020), Pangui (2018) y Saavedra y Camarena (2020) coinciden en señalar que las mujeres emprenden por la necesidad de tener un empleo remunerado y por las barreras impuestas por los techos de cristal ${ }^{4}$ (motivos extrínsecos), la aspiración de alcanzar logros, autorrealizarse e independizarse (motivos intrínsecos), la esperanza de encontrar un equilibrio entre sus actividades económicas y el cuidado de su familia (motivos trascendentales), la insuficiencia de ingresos económicos o la consecución del total de ingresos necesarios para asumir todos los gastos familiares (motivos económicos); se caracterizan por que no cuentan con formación empresarial o esta es mínima y lo mismo sucede con la experiencia laboral, y limitadamente han ocupado puestos directivos. Siguen la tendencia de administrar sus negocios desde su hogar, con lo que logran disminuir costos y riesgos, obteniendo la flexibilidad que requieren y buscan con el fin de compaginar sus actividades empresariales con las responsabilidades del hogar. López (2013) y Saavedra y Camarena (2020) afirman que la formación de las mujeres, en aspectos de negocios es principalmente empírica, adquirida en su participación en empresas familiares, muy pocas cuentan con conocimientos y herramientas tecnológicas; lo cual las deja en desventaja puesto que los estudios antecedentes han demostrado la importancia del conocimiento empresarial heredado en el buen desempeño de los negocios de las mujeres (López, 2013).

4- Se refiere a los obstáculos que la mujer encuentra para alcanzar los más altos niveles al interior de una organización y con ello una mejor posición económica (Camarena y Saavedra, 2018). 
En Latinoamérica las mujeres buscan tener su propia empresa particularmente en periodos de crisis económica; emprenden principalmente como una solución para generar su propio empleo, como una estrategia para sobrevivir (De Vita et al., 2014; Heller, 2010). Otras emprenden para llevar a cabo una idea innovadora y también lo hacen buscando alcanzar un nicho específico en el mercado o por una oportunidad encontrada en él, así como por el deseo de ser independientes financieramente (Machado et al., 2016). Las mujeres profesionales que emprenden lo hacen por encontrar barreras que les impiden desarrollarse dentro las organizaciones (techo de cristal) y/o porque desean tener una vida productiva económicamente sin relegar el cuidado de su familia, estas empresarias se caracterizan por contar con un nivel de estudios más elevado en comparación con los empresarios varones (De Vita et al., 2014; Heller, 2010; Machado, et al. 2016).

Así pues, la economía feminista teórica epistemológicamente busca construir nuevas configuraciones de análisis y la búsqueda de nuevos conceptos y categorías que fundamenten el estudio de la participación de la mujer en la economía considerando, la propia experiencia de las mujeres (Carrasco, 2006). Existe un número de investigaciones sobre estudios de género que han abordado el trabajo de las mujeres, bajo un tratamiento donde el hombre es el centro de todo o dentro de perspectiva analítica androcéntrica. La teoría neoclásica ha considerado a las mujeres "como esposas y madres dentro de una familia nuclear...; como trabajadoras menos productivas que los hombres en el trabajo... y como dependientes económicamente de sus maridos" (Carrasco, 2006, p. 31). Esta discusión sitúa en una posición económica y social más neutral a las mujeres, evitando el entendimiento tradicional de los análisis de género, dividiendo el mundo en dos espacios (hombres y mujeres), con reconocimientos distintos, donde precisamente se considera a las mujeres como jefas del hogar, enfoque que les permite acceder al mercado de trabajo, retomando el pensamiento feminista de Kate Sheppard sobre independencia económica de la mujer.

En este sentido, Sharma (2013) refiere que existen factores que empujan y jalan a las mujeres a una actividad económica; entre los factores que empujan (Push Factors) se encuentran elementos de necesidad tales como ingreso familiar insuficiente, insatisfacción con los salarios en el trabajo, dificultades para encontrar trabajo, la necesidad de flexibilidad en sus jornadas de trabajo por razones familiares (Pérez y Ceballos, 2018). Por otro lado, los factores que jalan (Pull Factors) son contemplados como la independencia, la auto realización, el deseo de riqueza, el poder y estatus social, la cooperación y apoyo familiar, y una red de contactos fuertes.

\subsection{Factores que influyen en el rendimiento económico de las mujeres}

Del conjunto de elecciones personales que las mujeres realizan en el ambiente empresarial, y que les permite decidir rechazar una promoción u ocupar puestos directivos o frenar el crecimiento de su propia empresa, esto es comúnmente denominado "techo de cemento" (BIRF, 2010; Chaves et al., 2013; Equal, 2010; Heller, 2010; Instituto de la Mujer, 2011; López-Diez, 2013; Pineda, 2014; Ribeiro, 2004; Saavedra, 2020; SELA, 2010).

Aunado a esto, también existen decisiones que motivan a una mujer a mantener la equidad entre sus responsabilidades empresariales y familiares, que constituyen obstáculos o límites para el desarrollo pro- 
fesional por tener que dedicar tiempo a ambas actividades. Ante esto, se presenta la interrogante sobre cuáles son las diferencias económicas de poder equilibrar lo laboral y lo familiar, entre mujeres jefas de familia asalariadas e independientes (empresarias), que explique su rendimiento.

Esto brinda la posibilidad de observar la existencia de diferentes "techos de cristal". En suma, a las providencias anteriores, se agrega coexistencia de lo que se denomina suelo pegajoso que consiste en las trabas que las mujeres profesionales enfrentan para obtener niveles más elevados de desarrollo al interior de las organizaciones (Torres y Pau, 2011). Si aquellas jefas de familia están determinadas por el grado educativo alcanzado y a su vez fija su orientación económica como su productividad, el techo de cristal estaría influido por el nivel escolar y su ruptura sería el efecto que produce aumentar un nivel educativo en su productividad (Teoría de capital humano).

Por otra parte, el tamaño de la empresa donde la mujer se desarrolla y se constituye el espacio de su competencia, generalmente, es el de las microempresas debido a las pocas barreras de ingreso (Brenes y Bermúdez, 2013; Camarena y Saavedra, 2016; Díaz y Jiménez, 2010; Escandón y Arias, 2011; Espino, 2005; García et al., 2011; Inmyxai y Takahashi, 2010; Powers y Magnoni, 2010; SELA, 2010), lo que entre otros aspectos, como son el limitado acceso a fuentes de financiamiento, un menor capital humano y el sector donde opera, estaría limitando su rendimiento (Afza, 2011, Saavedra, 2020). Dejando, como una incógnita si las diferencias en el mismo género es resultado del reparto desigual de capital humano, o derivadas de la distribución de responsabilidades propias del rol femenino reproductivo y familiar.

Si se parte del concepto sobre el jefe del hogar como aquella persona vinculada por sangre, matrimonio o adopción, a excepción de los empleados domésticos, que, además, es la autoridad central y responsable de todo lo referente al hogar, primordialmente de lo económico, cumpliendo el papel de proveedora de todas las necesidades del hogar y sus miembros; es posible traducir esta concepto hacia las jefas de hogar como mujeres que lidian entre responsabilidades familiares y económicas, que perciben ingresos realizando una actividad de forma independiente o asalariada, y formular las siguientes hipótesis.

$\mathbf{H}_{\mathbf{1}}$ : El rendimiento obtenido por jefas de hogar emprendedoras o independiente es inferior al obtenido por aquellas asalariadas.

$\mathbf{H}_{2}$ : Las mujeres que tienen la autoridad principal y económica en un hogar prefieren el emprendimiento a un trabajo subordinado, para equilibrar sus responsabilidades familiares y empresariales.

$\mathbf{H}_{3}$ : El techo de cristal de jefas de hogar mexicanas se determina por un capital humano con bajo nivel educativo.

\section{Metodología}

En esta investigación se aplica el proceso bietápico sugerido por Heckman et al., (2000 y 2001) con el fin de estimar los efectos de la aplicación de un tratamiento a las jefas de hogar. Para lo cual se calculan el "Average Treatment Effect", el "Effect of Treatment on the Treated", el "Local Average Treatment Effect" 
y el "Marginal Treatment Effect". Los datos con los que se trabajó se recopilaron de la Encuesta Nacional de Ingresos y Gastos de los Hogares (ENIGH) del 2016 (INEGI, 2017)5. En primer lugar, se extrajeron los datos a partir de una variable: Ingtrab (suma del ingreso obtenido por trabajo, subordinado independiente y otros trabajos si los hubiera).

Adicionalmente, identificando el sexo del jefe del hogar, se obtuvo el número total de jefas que ascendió a 15,271 registros, y con objetivo de distinguir aquellas que sus ingresos provenían principalmente de salarios y de actividades emprendedoras se elaboró un criterio de selección. Para tal propósito, se elaboró exprofeso dos ratios a partir de las variables de la base y como criterio para extraer la información, siempre y cuando los ingresos por trabajo subordinado e independiente fueran mayores al 75\%. En consecuencia, se eliminaron 2,555 registros de jefas que obtienen ingresos primordiales por otros trabajos que constituían el $17 \%$ del total.

De acuerdo con la descripción de la base de datos ENIGH 2016, los ingresos son trimestrales, netos y correspondientes al trabajo (Ingtrab). Por lo que la variable de la base "Ingtrab" es la suma de tres indicadores: a) Trabajo (subordinado), b) Negocio (independiente) y, c) otros_trab. Con lo que se pueden formular dos ratios y cumplir el criterio de $75 \%$, (Negocio/Ingtrab $>0.75$ y Trabajo/Ingtrab $>0.75$ ). Las definiciones de las variables de la base de datos son "Trabajo", monto de remuneraciones por trabajo subordinado (incluye sueldos, comisiones, aguinaldo, indemnizaciones y otros); "Negocio", monto de ingresos por trabajo independiente (ingresos monetarios o en especie); de "Otros_trab" como otros ingresos provenientes de otros trabajos que no sean la fuente principal de ingresos.

Con el objeto de determinar el salario por hora o su equivalente por actividades independientes de las jefas, la base presentó datos faltantes por lo que se eliminaron a 3,617 registros de 12,716. Finalmente, la muestra agrupo al 60\% de total de jefas del hogar, y en dos subgrupos con tratamiento (emprendedora), y sin él (asalariada); como consecuencia se obtuvo una muestra de 9,099 mujeres jefas de hogar, con la que se modela la elección de los individuos usando las variables: Educación, tamaño de la empresa, la existencia de niños menores de 12 años, total de integrantes, experiencia y salario (Ver cuadro 1).

5- Este trabajo tiene limitaciones dada la ENIGH empleada, donde el diseño de la fuente de datos no considera más factores adicionales que puedan ser estudiados, tanto en el diseño del instrumento y la recopilación de las preguntas de la encuesta. 


\begin{tabular}{|c|c|c|c|}
\hline \multicolumn{4}{|c|}{$\begin{array}{c}\text { Cuadro 1 } \\
\text { Parámetros, Descripción y Medición }\end{array}$} \\
\hline Variable & Descripción & Medición & Valoración \\
\hline \multirow{11}{*}{ Educa } & \multirow{11}{*}{$\begin{array}{l}\text { Educación formal del jefe } \\
\text { del hogar }\end{array}$} & 1. & Sin instrucción \\
\hline & & 2. & Preescolar \\
\hline & & 3. & Primaria incompleta \\
\hline & & 4. & Primaria completa \\
\hline & & 5. & Secundaria incompleta \\
\hline & & 6. & Secundaria completa \\
\hline & & 7. & Preparatoria incompleta \\
\hline & & 8. & Preparatoria completa \\
\hline & & 9. & Profesional incompleta \\
\hline & & 10. & Profesional completa \\
\hline & & 11. & Posgrado \\
\hline \multirow{12}{*}{ tam_emp } & \multirow{12}{*}{$\begin{array}{l}\text { Tamaño de la empresa en } \\
\text { base al número de traba- } \\
\text { jadores, donde desempe- } \\
\text { ñó su trabajo principal el } \\
\text { integrante del hogar, el } \\
\text { mes pasado. }\end{array}$} & 1 & De 1 persona \\
\hline & & 2 & De 2 a 5 personas \\
\hline & & 3 & De 6 a 10 personas \\
\hline & & 4 & De 11 a 15 personas \\
\hline & & 5 & De 16 a 20 personas \\
\hline & & 6 & De 21 a 30 personas \\
\hline & & 7 & De 31 a 50 personas \\
\hline & & 8 & De 51 a 100 personas \\
\hline & & 9 & De 101 a 250 personas \\
\hline & & 10 & De 251 a 500 personas \\
\hline & & 11 & De 501 a más personas \\
\hline & & 12 & No sabe \\
\hline Menores & Menores de 12 años & \multicolumn{2}{|c|}{$\begin{array}{l}\text { Cantidad de integrantes del hogar que tienen } 11 \text { o menos }<12 \\
\text { años de edad. }\end{array}$} \\
\hline Tot_integ & Total de Integrantes & \multicolumn{2}{|c|}{$\begin{array}{l}\text { Cantidad de personas que integran el hogar (no se consideran los } \\
\text { trabajadores del hogar sus familiares, ni a los huéspedes) }\end{array}$} \\
\hline Exper & $\begin{array}{l}\text { Experiencia del jefe de } \\
\text { hogar }\end{array}$ & \multicolumn{2}{|c|}{$\begin{array}{l}\text { Número de años de experiencia laboral, se calcula regularmente } \\
\text { por Exper = Edad - años de educación formal - } 6 \text {. }\end{array}$} \\
\hline Exper2 & $\begin{array}{l}\text { Experiencia al cuadrado } \\
\text { (Exper) } 2\end{array}$ & \multicolumn{2}{|c|}{$\begin{array}{l}\text { Se usa con el fin de demostrar la presencia de rendimientos decre- } \\
\text { cientes (dada la relación del capital humano con la productividad). }\end{array}$} \\
\hline L_Ing_hr & \multicolumn{3}{|c|}{$\begin{array}{l}\text { Logaritmo natural equivalente al salario por hora del jefe de hogar o logaritmo natural del ingre- } \\
\text { so por actividades independientes por hora del jefe del hogar. }\end{array}$} \\
\hline MI & \multicolumn{3}{|c|}{$\begin{array}{l}\text { Mujeres jefas de hogar: variable dicotómica, donde } 1 \text { es con tratamiento (independientes o } \\
\text { empresarias y } 0 \text { sin él (subordinadas) grupo de control. }\end{array}$} \\
\hline
\end{tabular}

Fuente. Elaboración propia. 


\subsection{Modelo de Estimación}

Para obtener una evaluación del efecto de una elección, se pueden desarrollar modelos econométricos que permitan predecir comportamientos de poblaciones o parámetros de interés en el estudio que se está realizando (Baker, 2000); si se considera que las jefas toman la decisión de participar en el emprendimiento no por voluntad propia, sino obligadas por las circunstancias que les toca vivir y que pueden ser atribuidos a múltiples factores ya explicados en la revisión la literatura. En este trabajo se resuelve el problema de sesgo y heterogeneidad aplicando el método propuesto por Heckman et al. (2000 y 2001), donde el problema surge cuando se extraen algunos individuos para integrar la muestra de un conglomerado, este diseño se llama muestreo bietápico.

Por lo que para analizar el resultado del tratamiento se tiene que medir la variable visible, calculada como la diferencia de $\mathrm{Y}_{1}-\mathrm{Y}_{0}$, lo que conduce a una ecuación 1 que representa la determinación de cada sujeto de participar en trabajo independiente, y aquellas que trabajan de forma subordinada (0). Conjuntamente, se consideran los rendimientos esperados de las jefas cuando varíen con el nivel educativo, el número de integrantes del hogar y menores, la experiencia, y el tamaño de empresa por los individuos con y sin tratamiento (subordinado e independiente), este tipo de situaciones no pueden ser medidas directamente (Willms, 2006).

La ecuación 1 simboliza la determinación de cada jefa en participar o no en actividades que le permitan obtener ingresos independientes, la cual es una variable latente $\mathrm{D}$, que puede explicarse con un conjunto de variables independientes contenidas en Z. Esto brinda la posibilidad de establecer una conexión entre el mecanismo de selección ( 1 y 0, con o sin tratamiento) y las variables independientes $\mathrm{X}$ incluidas en las ecuaciones (2) y (3). En este sentido, el resultado de la variable latente $\mathrm{D}$ es influida por el conjunto de variables $Z$, lo que es una limitación de supresión para una serie de variables independientes $\mathrm{X}$ que determinan a $\mathrm{Y}_{1}$ y $\mathrm{Y}_{0}$. Algunos investigadores (Arrazola y de Hevia, 2002; Austria-Carlos y VenegasMartínez, 2011, Austria-Carlos y Venegas-Martínez, 2018) que han empleado el método propuesto por Heckman et al. (2000 y 2001) para resolver un problema de sesgo mediante las siguientes ecuaciones:

$$
\begin{aligned}
& D=Z \theta+U_{D} \\
& Y_{1}=\beta_{1}+U_{1} \\
& Y_{0}=\beta_{0}+U_{0}
\end{aligned}
$$

en la que:

$$
\left(\begin{array}{l}
U_{D} \\
U_{1} \\
U_{0}
\end{array}\right) \sim N\left(0,\left[\begin{array}{ccc}
1 & \sigma_{1 D} & \sigma_{0 D} \\
\sigma_{1 D} & \sigma_{1}^{2} & \sigma_{10} \\
\sigma_{0 D} & \sigma_{10} & \sigma_{2}^{2}
\end{array}\right]\right)
$$

Con esta limitante, el modo de elección debe contener al menos una variable en $\mathrm{Z}$ que no esté contenida en X (véase Heckman y Vytlacil, 2001). A este respecto, Heckman et al. (2000, 2001) han propuesto 
realizar el proceso bietápico que consiste en estimar un modelo Probit el cual permite calcular una variable auxiliar denominada lambda $(\lambda)$, que tiene como función captar el efecto del sesgo de autoselección contenido en la variable latente $\mathrm{D}$. En una segunda etapa se realiza la estimación de las ecuaciones salariales ${ }^{6}$ empleando la variable $\lambda$, lo que permite corregir el sesgo originado por el seccionamiento, facilitando la obtención de datos más confiables.

Con objetivo de calcular los rendimientos considerando la ganancia log-salarial (MI) esperada para un conjunto de particularidades que se pueden observar en X, se calcularán cuatro parámetros que Heckman et al. (2000 y 2001) consideran en sus investigaciones y que permitirán resolver la hipótesis. Los parámetros son: a) el efecto medio del tratamiento (Average Treatment Effect, ATE), b) el efecto de tratamiento sobre lo tratado o rendimiento esperado por tratamiento (Effect of Treatment on the Treated, TT), c) el efecto local medio del tratamiento (Local Average Treatment Effect, LATE) y finalmente, d) el efecto marginal de tratamiento (Marginal Treatment Effect, MTE).

Definición y especificación de los Parámetros de Tratamiento de Interés

El efecto medio del tratamiento (ATE) aporta la ganancia esperada de participar en el programa para un individuo elegido al azar; se estima, en valores promedios, cuando se presentan un conjunto de variables observables contenidas en $\mathrm{X}=\mathrm{x}$. lo que facilitará entender si las mujeres con rendimientos cercanos a aquellas que trabajan de forma subordinada lo hacen por necesidad o deseo de riqueza y aquellos rendimientos lejanos, por responsabilidad con la familia. Esta estimación se realiza con la ecuación siguiente:

$$
\operatorname{ATE}(x)=E\left[Y_{1}-Y_{0} \mid X=x\right]=x\left(\beta_{1}-\beta_{0}\right)
$$

El segundo parámetro, el efecto del tratamiento en lo tratado (TT), es una ampliación del parámetro ATE, que es estimado con anterioridad, y se puede definir tradicionalmente como el rendimiento esperado para los individuos que participaron y recibieron el tratamiento voluntariamente. Entendiendo que el rendimiento esperado de estos $(D=1)$, se encuentra sujeto al conjunto de variables observables contenidas en $\mathrm{X}=\mathrm{x}$ y $\mathrm{Z}=\mathrm{z}$, contenidas en la siguiente ecuación:

$$
T T(x, z, D=1)=E\left[Y_{1}-Y_{0} \mid X=x, Z=z, D=1\right]=x\left(\beta_{1}-\beta_{0}\right)+\left(\rho_{1} \sigma_{1}-\rho_{0} \sigma_{0}\right) \frac{\varphi(z \theta)}{\Phi(z \theta)}
$$

Donde $\varphi($.$) simboliza la función de densidad de una variable aleatoria normal estándar, \Phi($.$) significa$ su función de distribución acumulada y 1 y 0 son los coeficientes de correlación entre U1 y UD, y U0 y UD, correspondientemente. Este parámetro mostrará que, efectivamente, el trabajo independiente de las mujeres posee el mayor efecto esperado en los rendimientos que el de las mujeres que trabajan de forma subordinada.

El tercer parámetro LATE, se describe como la ganancia de resultado esperada por los inducidos a

6- Ecuaciones típicamente, modeladas por el logaritmo de los ingresos como variable dependiente y como independientes la suma de variables como años de educación y claro, una función cuadrática de "años de experiencia potencia, véase: Mincer (1974). 
recibir tratamiento a través de un cambio en las variables observables incluidas en, los cuales impulsan a los individuos a recibir el programa. Este efecto se define a partir de un cambio de a con $\mathrm{Z}<\mathrm{z}^{\prime}$, en el que $\mathrm{z}$ y $\mathrm{z}^{\prime}$ son iguales excepto en el k-ésimo elemento. Este parámetro se entiende así:

$$
\begin{aligned}
& \operatorname{LATE}\left(D(z)=0, D\left(z^{\prime}\right)=1, X=x\right) \\
& \quad=E\left[Y_{1}-Y_{0} \mid D(z)=0, D\left(z^{\prime}\right)=1, X=x\right]=x\left(\beta_{1}-\beta_{0}\right)+\left(\rho_{1} \sigma_{1}-\rho_{0} \sigma_{0}\right) \frac{\varphi\left(\mathrm{z}^{\prime} \theta\right)-\varphi(\mathrm{z} \theta)}{\Phi\left(\mathrm{z}^{\prime} \theta\right)-\Phi(\mathrm{z} \theta)}
\end{aligned}
$$

La importancia de medir LATE es que permite simular impactos esperados (efectos locales) del programa, como resultado de cambios en los valores de variables incluidas, los que al compararse con el efecto ATE ayuda a establecer estrategias que permitan alcanzar eficientemente los objetivos del programa. La estimación de este parámetro se realiza principalmente sobre las variables que influyen de manera determinante en la decisión de los individuos para llevar a cabo o no el programa, variable de tratamiento.

El último parámetro, el efecto marginal del tratamiento (MTE), se estima para las preferencias de los individuos estudiados, que se encuentran en la frontera de participar, específicamente estos individuos no han recibido el tratamiento. Este componente es capaz de captar todos los factores inobservables () que de igual forma influyen de modo relevante en la variable latente, siendo linealmente independientes de las variables explicativas contenidas en $\mathrm{Z}$. Por lo que:

$$
\operatorname{MTE}\left(x, u_{D}\right)=E\left[Y_{1}-Y_{0} \mid X=x, U_{D}=u_{D}\right]=x\left(\beta_{1}-\beta_{0}\right)+\left(\rho_{1} \sigma_{1}-\rho_{0} \sigma_{0}\right) u_{D}
$$

\section{Estadística descriptiva correspondiente al grupo de tratamiento y de control}

Los resultados descriptivos (cuadro 2) explican las características entre las mujeres empresarias y aquellas subordinadas. Si el logaritmo natural del ingreso (L_Ing_hr) es exponente de un número $e$ de un argumento que constituye el ingreso por hora de una mujer, se observa que el valor medio es menor en las mujeres empresarias. Adicionalmente, el rango de la variable es similar en ambas mujeres, alrededor de 13, lo que apoya el planteamiento que existe una misma posibilidad de ingreso en los individuos.

En lo que respecta a sí la presencia de menores es una limitante para que una mujer realice actividades económicas, los datos mostraron que el valor medio es menor a la unidad. Lo anterior, no se repite en el total de integrantes en el hogar que mostró que el número promedio de sujetos es 3.32 y 2.51, por mujeres asalariadas y empresarias, respectivamente. Es indiscutible que el aumento en el número de integrantes puede influir en los ingresos o ser un reflejo de la necesidad como una consecuencia del ingreso familiar insuficiente. El número de integrantes del hogar funge como una variable que, con base en Alonzo, et al. (2004) y Austria-Carlos y Venegas-Martínez (2011), se considera que en los hogares que presentan elevada marginación se observa un número de miembros mayor, comparados con los hogares que presentan menor marginación. 


\section{Cuadro 2}

Estadísticos descriptivos de los grupos (1-9099)

\begin{tabular}{|c|c|c|c|c|c|}
\hline Variable & Observaciones & Media & Desviación Típica & Mínimo & Máximo \\
\hline \multicolumn{6}{|c|}{ Grupo de Control (0) Asalariadas } \\
\hline Menores & 7411 & 0.72 & 0.97 & 0.00 & 6.00 \\
\hline Educa & 7411 & 5.99 & 2.55 & 1.00 & 11.00 \\
\hline Exper & 7411 & 33.20 & 13.47 & 4.00 & 85.00 \\
\hline Exper2 & 7411 & $1,283.75$ & 994.08 & 16.00 & 7225.00 \\
\hline Tot_integ & 7411 & 3.32 & 1.73 & 1.00 & 18.00 \\
\hline L_Ing_hr & 7411 & 3.67 & 1.01 & -4.38 & 8.98 \\
\hline Tam_emp & 7411 & 4.26 & 3.48 & 1.00 & 12.00 \\
\hline \multicolumn{6}{|c|}{ Grupo de Tratamiento (1) Empresarias } \\
\hline Menores & 1688 & 0.53 & 0.90 & 0.00 & 7.00 \\
\hline Educa & 1688 & 4.38 & 2.33 & 1.00 & 11.00 \\
\hline Exper & 1688 & 43.37 & 16.79 & 5.00 & 98.00 \\
\hline Exper2 & 1688 & $2,163.46$ & $1,530.06$ & 25.00 & 9604.00 \\
\hline Tot_integ & 1688 & 2.51 & 1.50 & 1.0000 & 11.000 \\
\hline L_Ing_hr & 1688 & 2.39 & 1.54 & -4.83 & 8.68 \\
\hline Tam_emp & 1688 & 1.33 & 0.62 & 1.00 & 10.00 \\
\hline
\end{tabular}

Fuente: Elaboración propia con datos de la muestra.

Si se plantea a la variable Tam_emp como aquel espacio donde se desarrolla la actividad económica realizada por una mujer. Uno de los hallazgos surge de los estadísticos descriptivos donde la magnitud empresarial para una mujer asalariada es de una pequeña empresa (4.26) mientras que para mujer independiente es microempresa (1.33). Otro hallazgo que resulta importante es que la experiencia promedio de las mujeres asalariadas (33.2) es menor que el de aquellas que deciden emprender de forma independiente de 43.37 (Tabla 2).

La elección de realizar una actividad empresarial sobre la opción de trabajar de manera subordinada supone que existe una insatisfacción con los salarios en el trabajo, dificultades para encontrar trabajo, necesidad de flexibilidad en las jornadas de trabajo por razones familiares y también el deseo de autorrealización o auto dependencia económica, poder o status social (BIRF, 2010; Guzmán y Rodríguez, 2008; Pérez y Ceballos, 2018). La interrogante es si el motor de esta decisión está influido por la educación, puesto que la teoría de capital humano afirma que, al aumento de esta, aumenta la productividad. Así, el nivel educativo funge como un mecanismo transmisor de habilidades, competencias, conocimientos al individuo y su señalización de este, es el acceso, selección y filtro al tejido empresarial (Acevedo, et al., 2007; Pons, 2004). Los resultados estadísticos sobre el nivel escolar de mujeres asalariadas obtuvieron una media de 5.99 años o equivalente a secundaria completa, y para las empresarias o independientes el resultado fue 4.38 años o equivalentes a primaria completa (ver tabla 2). Lo que hace inferir que el capital humano determina el rendimiento de salarios (Mincer, 1974). 
La típica ecuación de regresión de Mincer, denominada "función de ingresos", contiene el logaritmo del ingreso a manera de variable explicada y la escolaridad y los años de experiencia a manera de variables explicativas (Acevedo, et al., 2007), y es por lo tanto este modelo estadístico propicio para esta investigación. Para resolver la hipótesis, y evitar la heterogeneidad inobservable que se produce al no disponer de alguna o algunas variables en el estudio dado su carácter de inobservabilidad, se corrigió el sesgo por autoselección utilizando la técnica biétapica, mediante un modelo Probit como primer paso y luego una ecuación salarial minceriana.

\section{Resultados del Modelo Econométrico}

En el cuadro 3, se resumen los resultados del modelo Probit, el cual ayudará a predecir la probabilidad de $\mathrm{Y}=1$ y no de $\mathrm{Y}=0$. La variable dependiente $M I$ está constituida por 1688 mujeres jefas de hogar con ingresos por actividades económicas independientes (empresaria) y 7411 mujeres jefas de hogar con ingresos subordinados o asalariados.

Cuadro 3

Modelo de Probit

\begin{tabular}{|c|c|c|c|c|c|}
\hline \multicolumn{7}{|c|}{ Modelo: Probit, usando las observaciones 1-9099 } & \\
\hline \multicolumn{7}{|c|}{ Coeficiente } & Desv. típica & & & \\
\hline Const & 0.296473 & 0.131875 & $* *$ & & \\
\hline Educa & -0.0375164 & 0.00895790 & $* * *$ & & \\
\hline Exper & 0.0104515 & 0.00527055 & $* *$ & & \\
\hline Exper2 & 0.000114753 & $6.22693 \mathrm{e}-05$ & $*$ & & \\
\hline Tot_integ & -0.136940 & 0.0157613 & $* * *$ & & \\
\hline Menores & 0.163842 & 0.0271976 & $* * *$ & & \\
\hline L_Ing_hr & -0.396878 & 0.0157519 & $* * *$ & & \\
\hline
\end{tabular}

Número de casos 'correctamente predichos' = 7709(84.7\%)

Variable dependiente: EM, desviaciones típicas basadas en el Hessiano.

${ }^{\star} \mathrm{P}(\mathrm{t})$ significativo a $.05{ }^{* \star} \mathrm{P}(\mathrm{t})$ significativo a $.01,{ }^{* *} \mathrm{P}(\mathrm{t})$ significativo a .001

Con la ecuación resultante del modelo Probit se estima el término de lambda $(\lambda)$, mismo que mide el efecto por autoselección, corrigiendo el sesgo incurrido en la estimación de la regresión de las ecuaciones salariales mincerianas por MCO. En suma, la bondad de ajuste se basa en el porcentaje de observaciones proyectadas correctamente que fue de $84.7 \%$. De la interpretación de los coeficientes de las variables significativas se infiere que si, es mayor que cero decimos que cuando, aumenta, la probabilidad de que Y sea 1 aumenta.

Los resultados encontrados fueron que la educación, total de integrantes, menores y el logaritmo natural del ingreso por hora fueron variables significativas en la probabilidad de ser una mujer independiente o empresaria. Ciertamente, los efectos marginales de las variables expresan: 1) la educación tiene un efecto negativo, al aumento de nivel escolar de $-0.83 \%$ sobre la probabilidad, 2) el aumento en el nú- 
mero de menores, posee un efecto positivo de $4.01 \%, 3$ ) al incrementar el número total de integrantes, el efecto que produce es $-2.85 \%$, y finalmente, 4 ) al aumento en una unidad el ingreso ( $\left.L \_I n g \_h r\right)$, el efecto que obtiene es $-7.10 \%$.

Del mecanismo de elección para responder a las hipótesis planteadas, se infiere que las mujeres realizan la elección de emprendimiento independiente palpablemente por la necesidad de equilibrar su responsabilidad familiar y empresarial, por el efecto positivo de menores, y el negativo en $L \_I n g \_h r$. Por su parte, el aumento de responsabilidades con el hogar, el ítem tot_integ, reduce la probabilidad y si se adiciona el efecto educación, se tiene que las mujeres enfrentan problemas en la búsqueda de puestos de trabajo con mayores competencias-remuneración, filtros de acceso a puestos, o que para emprender de forma de independiente presenta menores barreras de ingreso que para acceder a una actividad económica como asalariada, dado su nivel de educación básica alcanzado por los sujetos de tratamiento (jefas empresarias).

Para cumplir con la restricción de exclusión (ver modelo de estimación), la forma de elección debe considerar al menos un elemento en Z (ecuación de probabilidad) que no esté incluida en X (ecuación MCO) (Heckman y Vytlacil, 2001), por lo que en la ecuación salarial la variable tam_emp es incluida y permite observar si el tamaño de la empresa influye en los ingresos de las jefas de hogar. Así también, es posible incluir las variables independientes de la ecuación de interés, la cual deberá contener al menos una variable que resulte determinante en el proceso de formar parte o no de la muestra, la cual a su vez no debe ser relevante para determinar si se es o no empresaria (variable dependiente) (Sánchez et al., 2011).

En el cuadro 4, se presenta la ecuación salarial minceriana para los grupos de tratamiento y control. Lo anterior, con el fin de enfatizar el rol de $\lambda$, y a partir de su significación estadística, siendo posible así concluir que la heterogeneidad y la autoselección tienen influencia en la diferencia salarial entre las mujeres independientes y subordinadas. La ecuación salarial minceriana o función de ingresos mide la influencia de un conjunto de variables en los ingresos de las jefas de hogar. Se observa que únicamente la variable Tam_emp no fue significativa, sin embargo, no se puede inferir aún ni descartar si el tamaño empresarial explica la competencia en la que se desarrollan las jefas de hogar, particularmente las independientes, por las pocas barreras de acceso al mercado que poseen las empresas de menores dimensiones. Por su parte, la variable lambda presenta un resultado significativo estadísticamente, lo que deja en evidencia que de no controlarse la heterogeneidad y la autoselección los estimadores de MCO resultarían sesgados y los parámetros de interés serían incorrectos.

\section{Cuadro 4}

\begin{tabular}{|c|c|c|c|c|}
\hline \multicolumn{7}{|c|}{ Ecuación salarial minceriana } \\
\hline & Coeficiente & Desv. Típica & & \\
\hline Const & 4.63511 & 0.0401735 & $* * *$ & \\
\hline Educa & -0.0170478 & 0.00263799 & $* * *$ & \\
\hline Exper & -0.0111073 & 0.00144169 & $* * *$ & \\
\hline Exper2 & 0.000697257 & $1.87371 \mathrm{e}-05$ & $* * *$ & \\
\hline Tot_integ & -0.0719016 & 0.00337621 & $* * *$ & \\
\hline Tam_emp & 0.00149006 & 0.00162673 & & \\
\hline Lambda( (1) & -8.02792 & 0.0410785 & $* * *$ & \\
\hline
\end{tabular}

Variable dependiente: 1 _ing_hr., usando las observaciones 1-9099 (Incluyen con o sin tratamiento) R-cuadrado: 0.848844 , R-cuadrado corregido 0.848744. 
Finalmente, el último paso del modelo econométrico es estimar por separado las ecuaciones salariales incluyendo la variable lambda $\lambda$, para cada uno de los grupos, de tratamiento (1) y de control (0) (ver cuadro 5).

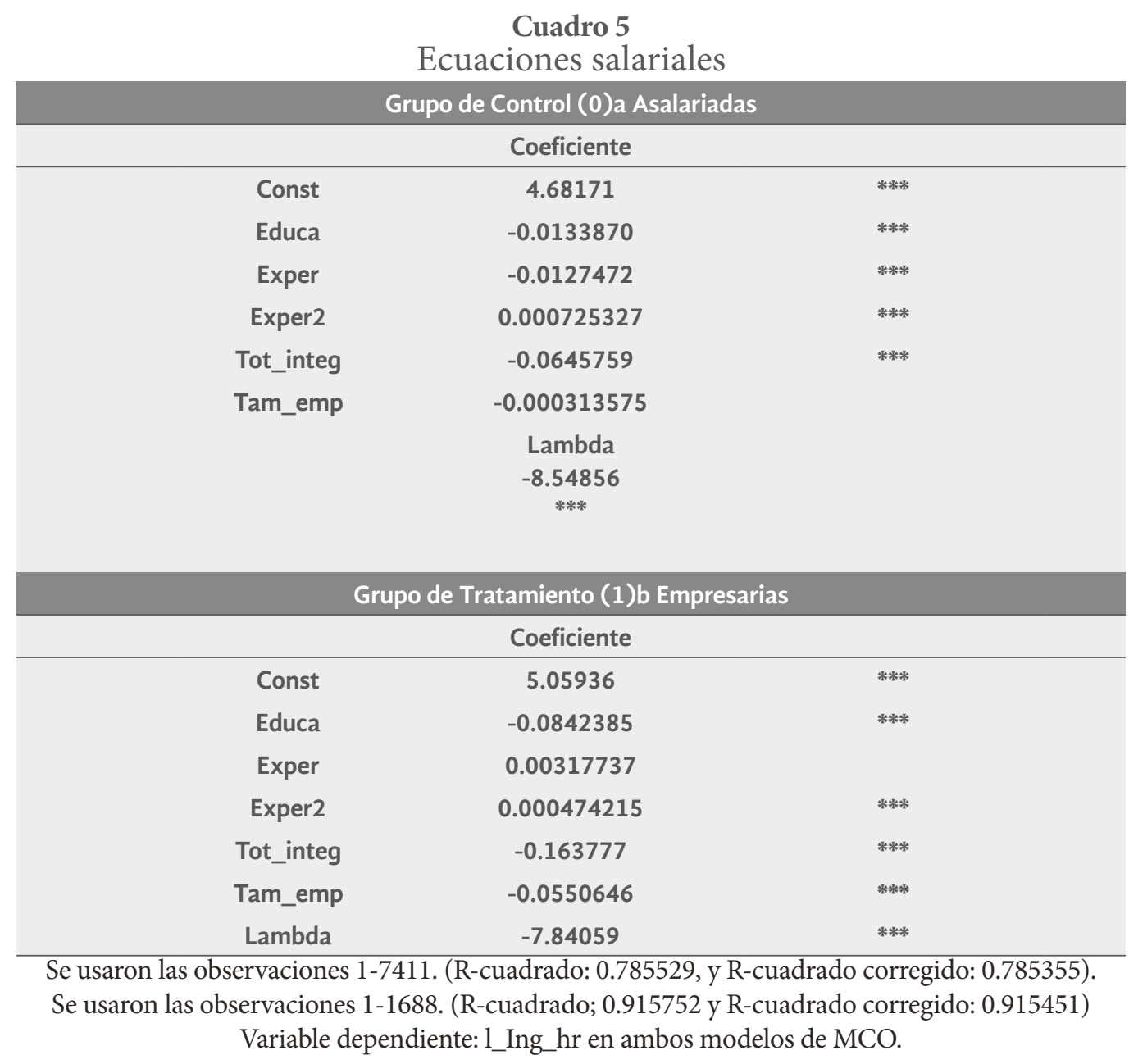

De las ecuaciones salariales obtenidas, se toman los valores estimados que corresponden a la variable dependiente l_Ing_hr con los que se calcula el efecto promedio de las mujeres jefas del hogar en actividades económicas, con (independientes) y sin tratamiento (asalariadas), en México respecto al ingreso por hora de los sujetos para así determinar los rendimientos. El cuadro 5 proporciona la base para calcular los efectos de los tres parámetros de interés: ATE, TT, y MTE. En cuanto al tratamiento se realizan las estimaciones de la variable dependiente, para cada ecuación salarial y se obtienen las estimaciones para determinar el efecto medio del tratamiento (ATE, Average Treatment Effect), mediante la aplicación de la ecuación 4, con los valores promedios obtenidos, permite tener como resultado el rendimiento esperado para cualquier individuo, dado un conjunto de variables observables incluidas en $\mathrm{X}=\mathrm{x}$, se obtuvo un valor de -1.04, esto es que las mujeres que realizan actividades económicas de forma independiente tienen un crecimiento exponencial negativo 1.04 veces menor esperado, que para una mujer elegida al azar de las mujeres subor- 
dinadas (cuadro 6), con estos resultados se acepta $\mathbf{H}_{1}$ y se concluye que las mujeres empresarias obtienen menos rendimientos que las mujeres asalariadas, lo cual también era evidente en los resultados descriptivos donde el promedio de ingresos de una asalariada resultó superior al de una empresaria.

Cosa parecida sucede con el segundo parámetro, el efecto de tratamiento sobre lo tratado (Effect of Treatment on the Treated, TT), este es el rendimiento promedio del tratamiento para los individuos que realmente seleccionan el tratamiento, es decir para aquellas jefas que eligieron ser económicamente independientes, obtuvo un valor de -0.95. Este resultado, confirma que no existe deseo de riqueza por las mujeres al elegir esta forma de actividad económica, lo que es coincidente con la literatura previa que afirma que la mujer busca el equilibrio familiar y económico (BIRF, 2010; De Vita et al., 2014; Escamilla, et al., 2013; Guzmán y Rodríguez, 2008; Heller, 2010; López, 2013).

En referencia al efecto marginal del tratamiento (Marginal Treatment Effect, MTE), se estima para las preferencias de los individuos que están en el límite de participar en el programa, sin haber tomado aún la decisión de hacerlo o no. La importancia de este parámetro es en la medida que se aproxima a 0, aumenta la probabilidad de que decidan participar en el programa. En este estudio, al contar con rendimientos negativos que evidentemente afectan el programa (elección) supone una decisión de no participar, dado que -1.04 veces es el rendimiento de las mujeres al participar en actividades independientes.

Todas estas observaciones permiten inferir que la flexibilización del trabajo y la asunción de responsabilidades familiares son factores más importantes sobre el deseo de riqueza de la mujer, con lo cual la motivación únicamente responde a la necesidad de equilibrar sus responsabilidades familiares y empresariales. Lo anterior implica que la mujer construye para sí misma un "techo de cemento" (BIRF, 2010; Chaves et al., 2013; Equal, 2010; Heller, 2010; Instituto de la Mujer, 2011; López-Diez, 2013; Pineda, 2014; Ribeiro, 2004; Saavedra, 2020; SELA, 2010), el cual representa un límite al crecimiento de la empresa, con tal de no descuidar sus responsabilidades familiares.

El resultado anterior, permite aceptar $\mathbf{H}_{2}$, y se concluye que las mujeres jefas de familia mexicanas prefieren emprender para equilibrar sus responsabilidades familiares y empresariales.

\section{Cuadro 6}

Efectos del programa de educación superior en los ingresos

\begin{tabular}{|cc|}
\hline Parámetros & Valores absolutos \\
\hline ATE & -1.04 \\
TT & -0.95 \\
MTE & -1.01 \\
\hline
\end{tabular}

Fuente: Elaboración propia, a partir de los datos de ENIGH, 2016.

Otro parámetro de interés es el efecto LATE, es decir el rendimiento esperado del programa atribuible a cambios en los factores visibles contenidos en . 7 . Para efectos de este trabajo, los efectos LATE representan los rendimientos esperados de las mujeres independientes cuando se hace aumentar en una unidad los valores medios en la educación de la jefa de hogar, en el número total de integrantes, y 
menores, es decir, permitiendo así estimar los efectos esperados (efectos locales) del programa como resultado de cambios en los valores de algunas variables independientes.

\section{Cuadro 7}

Efecto LATE en el grupo de tratamiento, mujeres independientes

\begin{tabular}{|c|c|}
\hline Variable $(\mathrm{Xi})$ & Valores absolutos \\
\hline Educa & -0.82 \\
\hline Tot_integ & -0.62 \\
\hline Menores & -1.32 \\
\hline
\end{tabular}

Fuente: Elaboración propia, a partir de los datos de ENIGH, 2016.

Los resultados de LATE (cuadro 7) a partir del efecto local de la variable Educa, conducen a la aceptación de $\mathbf{H}_{3}$ y se infiere que, para las mujeres empresarias mexicanas, al aumento de educación aumenta su rendimiento, esto es, reduce el impacto de tal emprendimiento, al poder acceder a un trabajo asalariado, y así romper el techo de cristal, puesto que en el análisis descriptivo se observó que las empresarias contaban con un menor nivel educativo que las asalariadas. Dentro de los efectos locales, LATE, el que más impacto tiene sobre su rendimiento es la variable Menores que se traduce en el aumento en el número de integrantes y se infiere que el aumento de responsabilidades familiares limita el crecimiento de sus rendimientos (techo de cemento).

El aventurarse en actividades económicas con un mayor número de personas en el hogar mostró un efecto también negativo a disminuir el rendimiento (-0.62), aunque es preciso mencionar que el impacto en rendimiento de una mujer empresaria es menor. Evidentemente, los menores y el total de integrantes en un hogar son un motivo para buscar la flexibilidad en sus jornadas de trabajo por razones familiares. Esto es coincidente con Ribeiro (2004) que señala que cuando una mujer desarrolla una actividad económica aparte de sus labores del hogar, debe estar dispuesta a asumir una doble jornada laboral.

\section{Discusión}

Si bien la agenda de la administración social ha buscado aminorar las desigualdades de género, la falta de un enfoque sobre el curso de vida de las mujeres ha dificultado la vinculación entre los programas de política social y las necesidades de las mujeres jefas de familia (Altamirano et al., 2020). En lo que se refiere a la igualdad de oportunidades entre mujeres en el proceso de selección y superación personal, los resultados revelan la ausencia de iniciativas públicas y privadas para la protección de la mujer trabajadora e independiente, es decir, como lo han planteado Jiménez y Hernández (2020) las dificultades persisten ante la falta de prácticas organizacionales que fomenten la conciliación laboral y familiar como, por ejemplo, la organización de los horarios de trabajo para las mujeres embarazadas, trabajo a tiempo parcial, reducir la doble jornada y mejoramiento de las condiciones de trabajo.

Esta situación debe ser atendida con urgencia pues la Organización Internacional de Trabajo (OIT), en los objetivos de agenda 2030 de Desarrollo Sostenible, insta a promover un crecimiento económico sostenido, inclusivo y sostenible, el pleno empleo productivo y el trabajo decente, así como la igualdad de 
género (Agenda 2030 de Desarrollo Sostenible, 2020). Lo anterior, es necesario que sea instrumentado en México a través de las políticas públicas, para que las mujeres jefas de familia independientes cuenten con un trabajo decente, al cual no han tenido acceso por la discriminación a su rol social de género; así como, aumentar las oportunidades de acceso a un mejor trabajo remunerado dado su nivel educativo, en el caso de las mujeres asalariados, y en el caso de las mujeres amas de casa ante la imposibilidad de disminuir la invisibilidad de su trabajo (Mejía et al, 2020 y Pangui, 2018). Dado que mientras, esta situación no sea resuelta tendremos una asignatura pendiente para alcanzar los objetivos de la Agenda 2030.

\section{Conclusión}

Esta investigación buscó determinar los factores que influyen en las mujeres mexicanas para emprender su negocio y balancear sus actividades con los roles tradicionales reproductivos y de responsabilidad en el hogar. Primeramente, la encuesta ENIGH, permitió observar parcialmente a las mujeres emprendedoras bajo la peculiaridad de jefas de hogar, es decir aquellas mujeres que lidian entre responsabilidades familiares y económicas, y son la autoridad en un hogar. Los resultados empíricos obtenidos mostraron que el rendimiento de una jefa de hogar al elegir ser independiente económicamente (empresaria) posee una particularidad de trabajo complementario evidenciado por los efectos de tratamiento que, al participar en esta actividad económica, el crecimiento de los ingresos es negativo comparado por la elección de ser jefa de familia asalariada. Indiscutiblemente, la presencia de los menores refleja su papel reproductivo e incluso exclusivo de la mujer, pero resalta el sacrificio profesional y económico que significa dicha opción. Lo anterior, es explicado por la teoría de la Identidad Performativa, como resultante de que la visión de la mujer independiente sobre la actividad emprendedora no es necesariamente el dinero; el motivador más fuerte es la percepción de una flexibilización de los tiempos que la estructura laboral dependiente no brinda, al contar con una estructura diseñada por hombres y para ellos. En este sentido, se hace hincapié en que la identidad emprendedora de la mujer:

Se construye y reconstruye a sí misma desde una identidad en constante disputa en su relación con el entorno y en la multiplicidad de tareas y roles que debe jugar diariamente como mujer, atravesada por su condición de clase. No se es sólo emprendedora, también se es madre, vecina, esposa, cliente, jefa, amiga y vecina. (Astorga, 2019, p. 10)

El efecto LATE, básicamente reafirmó que la mujer asalariada y jefa de familia presenta mayores rendimientos en sus ingresos que aquellas mujeres jefas con ingresos independientes como empresarias. Es de destacar el hallazgo de que las jefas emprendedoras tienen un nivel educativo medio de primaria completa, inferior a la media de la jefa asalariada, lo que permite inferir que cuando la mujer mexicana incrementa su nivel educativo, esto representa romper un "techo de cristal". Lo anterior, es palpable en el efecto LATE de la variable educa, con un valor negativo de -0.82, es mayor que el -1.04 del efecto ATE, revelando que al aumento de nivel educativo produce un efecto positivo en el rendimiento, lo que significa que la inversión en alcanzar un mayor nivel educativo mejora su nivel de vida.

Sin embargo, también surgen interrogantes de que, si esta limitante o falta de interés por aumentar el 
nivel educativo es producto de factores de no contar con la credencialización, (señal en la teoría de capital humano), por los filtros de selección a puestos de trabajo subordinado, por no contar con las competencias propias del nivel educativo, o por no existir puestos vacantes en el mercado laboral, etc. Estos resultados sugieren estar en presencia de diferentes techos de cristal para la mujer jefa de familia mexicana que por roles tradicionales que desmotivan el ingreso de las mujeres al mundo empresarial, puesto que el costo económico para una mujer sobre elegir contratarse o autoemplearse, es más de naturaleza de necesidad qué por una búsqueda de riqueza, esto queda demostrado dada la diferencia mínima entre ATE y MTE.

Lo anterior, se valida con la ecuación minceriana resultante (Tabla 4) de las mujeres por ingresos del trabajo (asalariado e independiente) en la cual se observa que los coeficientes significativos donde el signo es negativo fueron Educa, Exper, y Tot_integ, entendiendo que al aumento de estos tiende a reducir el crecimiento del ingreso de la mujer mexicana, Confirmándose lo referido por Sheehy (1986, p. 358): "La mayoría de las mujeres se sienten obligadas a escoger entre el amor y los hijos o el trabajo y la realización".

Por último, los efectos que producen la presencia de menores y el número total de integrantes de un hogar en los rendimientos para la jefa de hogar mexicano dejan futuras hipótesis de investigación sobre la existencia de limitantes institucionales que vigorizan el techo de cemento, como lo es la falta de esquemas de apoyo, creación de redes en pro de la mujer empresaria, fuentes accesibles para recursos y otros que mejoren su costo de oportunidad.

\section{Referencias}

Acevedo, C., Montes, I., Maya, V., González, V., y Mejía, B. (2007). Capital humano: una mirada desde la educación y la experiencia laboral. Cuadernos de investigación, (56), 1-31. https://publicaciones. eafit.edu.co/index.php/cuadernos-investigacion/article/view/1287/1166

Afza, N. (2011). The effect of owner's gender and age to firm performance: A review on Malaysian public listed family businesses. Journal of global business and economics, 2(1), 1-13.

Agenda 2030 de Desarrollo Sostenible. (2020). Organización Internacional de Trabajo website: http:// www.oit.org/global/topics/sdg-2030/lang--es/index.htm

Alonzo, R., Balisacan, A., Canlas, D., Capuno, J., Clarete, R., Danao, R., y Bautista, M. S. (2004). Population and poverty: the real score. UP School of Economics Discussion Papers 200415, University of the Philippines School of Economics. http://www.econ.upd.edu.ph/dp/index.php/dp/article/view/120/117

Altamirano, M., Morayta, G. C., y Mabire, B. (2020). Política social e igualdad de género en México, 2012-2018. Foro Internacional, 60(2). 755-790. https://doi.org/10.24201/fi.v60i2.2738

Arrazola, M., y de Hevia, J. (2002). Estimación de los efectos de un" tratamiento": una aplicación a la educación superior en España. Papeles de trabajo del Instituto de Estudios Fiscales. Serie economía, 25 (02), 3-22. https://www.ief.es/docs/destacados/publicaciones/papeles_trabajo/2002_25.pdf

Astorga, P. S. S. (2019). Mujeres emprendedoras: Abordaje desde la Teoría de la Identidad Performativa. Revista Estudios Feministas, 27(3). https://doi.org/10.1590/1806-9584-2019v27n354270

Austria-Carlos, M. A., y Venegas-Martínez, F. (2011). Rendimientos privados de la educación superior en México en 2006. Un modelo de corrección del sesgo por autoselección. El trimestre económico, 78(310), 441-468. 
Austria-Carlos, M. A., Venegas-Martínez, F., y Pérez Lechuga, G. (2018). Diferencias por género en la tasa de ganancia salarial de la educación superior y posgrado en México. Papeles de población, 24(96), 157-186. http://dx.doi.org/10.22185/24487147.2018.96.18

Baker, J. L. (2000). Evaluación del impacto de los proyectos de desarrollo en la pobreza: Manual para profesionales. Banco Mundial. http://documents.worldbank.org/curated/en/974581468278042080/Evaluacion-del-impacto-de-los-proyectos-de-desarrollo-en-la-pobreza-manual-para-profesionales

BIRF (2010). Mujeres empresarias barreras y oportunidades en el Sector Privado formal en América Latina y el Caribe. Banco Mundial.

Brenes, L. y Bermúdez, L. (2013). Diferencias por género en el emprendimiento empresarial costarricense. Tec Empresarial, 7(2) 19-27. https://doi.org/10.18845/te.v7i2.1510

Camarena-Adame, M. E. y Saavedra-García, M. L. (2018). El techo de cristal en México. Revista de estudios de género, la ventana, 5(47), 312-347. https://doi.org/10.32870/lv.v5i47.6680

Camarena-Adame, M. E., y Saavedra-García, M. L. (2016). Un estudio de las PYME lideradas por mujeres en Latinoamérica. Revista Universitaria Ruta, 18(1), 1-27. https://revistas.userena.cl/index. $\mathrm{php} / \mathrm{ruta} /$ article/view/789

Carrasco, C. (2006). La economía feminista: Una apuesta por otra economía. En M. J. Vara, Estudios sobre género y economía (Vol. 15. Pp. 29-62). Ediciones Akal.

Chaves, M., Valenciano, A., Vega, G. y Ortiz, G. (2013). Mejorando la competitividad de las microempresas en la región brunca de Costa Rica. Revista Ciencias Sociales, 1(139), 137-149.

De Vita, L.; Mari, M. y Poggesi, S. (2014). Women entrepreneurs in and from developing countries: Evidences from the literature. European Management Journal, 32(3) 451-460. https://doi.org/10.1016/j. emj.2013.07.009

Díaz García, M. y Jiménez Moreno, J. (2010). Recursos y resultados de las pequeñas empresas: nuevas perspectivas del efecto género. Cuadernos de economía y Dirección de la empresa, 13(42), 154-76. https://doi.org/10.1016/S1138-5758(10)70006-2

Equal (2010). Guía para la incorporación de políticas de igualdad de género en las PYMES. Unión Europea. http://www.surt.org/accions/guia_delta_igualdad_pimes.pdf

Escamilla, D.; Parra, Y.; Sepúlveda, M. y Vázquez, V. (2013). Familias monoparentales, madres solteras jefas de hogar. Investigación Cualitativa, 2(15), 1-17. http://www.ubiobio.cl/miweb/webfile/media/357/Articulos/Art\%C3\%ADculo-\%20Familias\%20monoparentales.pdf

Escandón, D. M. y Arias, A. (2011). Factores que componen la competitividad de las empresas creadas por mujeres y las relaciones entre ellos. Cuadernos de administración, 24(42),165-181.

Espino, A. (2005). Un marco de análisis para el fomento de las políticas de desarrollo productivo con enfoque de género. Naciones Unidas-CEPAL. https://repositorio.cepal.org/handle/11362/5798

Farias, S. ; Salazar, M. ; Monjardín, F. y Calleja, N. (2014). Jefas de familia en México : Caracterización y rasgos instrumentales-expresivos. Revista Mexicana de Investigación en Psicología, 6(1), 87-96.

García, B. y De Oliveira, O. (2005). Mujeres jefas de hogar y su dinámica familiar. Papeles de población, 11(43), 29-51.

García, M., García, D. y Madrid, A. (2012). Caracterización del comportamiento de las Pymes según el género del gerente: un estudio empírico. Cuadernos de Administración, 28(47), 37-52. 
González, P.; Rangel, Y. y Hernández, E. (2020). Retos en la prevención del embarazo adolescente subsiguiente, un estudio desde la perspectiva de madres adolescentes. Saúde e Sociedade, 29(3). https:// doi.org/10.1590/s0104-12902020181032

Guzmán, J. y Rodríguez, M. (2008). Comportamiento de las mujeres empresarias: una visión global. Revista de Economía Mundial, (18), 381-392.

Heckman, J. J., Tobias, J. L. y Vytlacil, E. (2000). Simple estimators for treatment parameters in a latent variable framework with an application to estimating the returns to schooling. Working paper 7950. National Bureau of Economic Research. https://www.nber.org/papers/w7950.pdf

Heckman, J., Tobias, J. L., y Vytlacil, E. (2001). Four parameters of interest in the evaluation of social programs. Southern Economic Journal, 68(2), 211-223. https://www.jstor.org/stable/1061591?seq=1

Heckman, J., y Vytlacil, E. (2001). Identifying the role of cognitive ability in explaining the level of and change in the return to schooling. Review of Economics and Statistics, 83(1), 1-12. https://www.jstor. $\mathrm{org} /$ stable/2646685? seq=1

Heller, L. (2010). Mujeres emprendedoras en América Latina y el Caribe: realidades, obstáculos y desafíos. Naciones Unidas-CEPAL, División de asuntos de género. https://repositorio.cepal.org/bitstream/ handle/11362/5818/1/S0900676_es.pdf

INEGI (2017). Encuesta Nacional de Ingresos y Gastos de los Hogares (ENIGH) 2016. Instituto Nacional de Estadística Geografía e Informática. https://www.inegi.org.mx/programas/enigh/nc/2016/

Inmyxai, S. y Takahashi, Y. (2010). Performance contrast and its determinants between male and female headed firms in Lao MSMEs. International Journal of business and management, 5(4) 37-52. https:// doi.10.5539/ijbm.v5n4p37

Instituto de la Mujer (2011). Los recursos y resultados empresariales: Una perspectiva de Género. Universidad de Castilla la Mancha. http://www.inmujer.gob.es/areasTematicas/estudios/serieEstudios/ docs/recursosResultadosEmpresariales.pdf

Jiménez, A. y Hernández, A. (2020). Percepción de equidad de género y equilibrio trabajo-familia en trabajadores pertenecientes a empresas públicas y privadas de Chile. Ciencias Psicológicas, 14(2). http://dx.doi.org/10.22235/cp.v14i2.2201

Lázaro, R., Zapata M. y Martínez, B. (2007). Jefas de hogar: cambios en el trabajo y en las relaciones de poder. Política y Cultura, (28), 194-218. https://www.redalyc.org/articulo.oa?id=26702809

Lechuga, J.; Ramírez, G. y Guerrero, M. (2018). Educación y género. El largo trayecto de la mujer hacia la modernidad en México. Economía UNAM, 15(43), 110-139. http://www.scielo.org.mx/pdf/eunam/v15n43/1665-952X-eunam-15-43-110.pdf

López, E. (2013, 28-31 de mayo). Las mujeres en empresas, profesiones y ocupaciones no tradicionales: La experiencia de Puerto Rico. Global Conference on Business and Finance, Proceedings, Costa Rica, 8(2), 1564 -1574. https://www.theibfr.com/wp-content/uploads/2016/06/ISSN-1941-9589-V8-N2-2013-1.pdf

López-Diez, A. (2013). Emprender: Una perspectiva de género. Universida de da Coruña, Servizo de Publicacións. https://www.udc.es/export/sites/udc/oficinaigualdade/_galeria_down/documentos/EMPRENDER_UNA_PERSPECTIVA_DE_GENERO_reducido.pdf_2063069299.pdf

Machado, H. P. V., Gazola, S., Dos Santos F. J. y Anez, M. E. M. (2016). Women Entrepreneurs: Reasons and Difficulties for Starting in Business. Revista de Administração Mackenzie, 17(3), 15-38. https:// doi.org/10.1590/1678-69712016/administracao.v17n3p15-38 
Mejía, O. G., Proaño, M. F. y Murillo, E. P. (2020). Características del perfil emprendedor de las madres solteras del noroeste de la ciudad de Guayaquil. Revista Investigación y Negocios, 13(21), https://doi. org/10.38147/invneg.v13i21.86

Mincer, J. (1974). Schooling, Experience, and Earnings. The National Bureau of Economic Research (NBER). https://www.nber.org/books/minc74-1

OIT (2015). La Mujer en la gestión empresarial. Cobrando impulso. Oficina Internacional del Trabajo. https://www.ilo.org/wcmsp5/groups/public/---dgreports/---dcomm/---publ/documents/publication/wcms_335674.pdf

Pangui, F. T. (2018). Emprendedoras, empresarias y empoderadas: reflexiones en torno al programa Mujeres Jefas de Hogar del Servicio Nacional de la Mujer y Equidad de Género de Chile. Revista Latinoamericana de Antropología del Trabajo, 2(4), 1-26. http://www.ceil-conicet.gov.ar/ojs/index.php/ lat/article/viewFile/385/292

Pérez, J. y Ceballos, G. (2018). Dimensionando la precariedad laboral en México de 2005 a 2015, a través del modelo logístico ordinal generalizado. Noésis, Revista de Ciencias Sociales y Humanidades, 28(55), 110-138. http://dx.doi.org/10.20983/ noesis.2019.1.6

Pineda Duque, J. A. (2014). Emprendimiento y género: el caso de la industria de la belleza en Bogotá. Sociedad y economía, (26), 239-272.

Pons B., M. (2004). Determinación Salarial: Educación y Habilidad. Análisis teórico y Empírico del caso español. [Tesis doctoral, Universitat de Valencia. Facultat de Ciències Econòmiques i Empresarials. Departament d'Anàlisi Econòmica Repositorio Institucional UV. http://roderic.uv.es/handle/10550/15154

Powers, J. y Magnoni, B. (2010). Dueña de tu propia empresa: Identificación, análisis y superación de las limitaciones a las pequeñas empresas de las mujeres en América Latina y el Caribe. Fondo Multilateral de Inversiones, BID. https://publications.iadb.org/es/publicacion/13690/duena-de-tu-propia-empresa-identificacion-analisis-y-superacion-de-las

Ribeiro, M. (2004). Relaciones de género: equilibrio entre las responsabilidades familiares y profesionales. Papeles de Población, 10(39), 219-234.

Rodríguez Fernández, A., Sánchez Santa-Bárbara, E. y Estévez López, E. (2011, 16-18 de febrero). Las mujeres emprendedoras en las comunidades indígenas. En F. J. García Castaño y N. Kressova. (Coords.). Actas del I Congreso Internacional sobre Migraciones en Andalucía (pp. 59-66), Granada, Instituto de Migraciones. https://dialnet.unirioja.es/servlet/articulo? codigo=4030586

Saavedra García, M.L. (2020). La competitividad en MIPYMES dirigidas por mujeres en la Ciudad de México. Ciencias Administrativas, 8(15,) 51-64. https://doi.org/10.24215/23143738e055

Saavedra García, M. L. y Camarena Adame, M. E. (2020). SMEs led by women and training: an exploratory study. Small Business International Review, 4(2), 1-17. https://doi.org/10.26784/sbir.v4i2.270

Sánchez, S.; Ravelo, P. (2013). Cultura de la violencia en el contexto de la vida cotidiana de la clase obrera en las maquiladoras de Ciudad Juárez. El Cotidiano, (182), 41-50. http://www.redalyc.org/articulo. oa?id=32529942005

Sánchez, F. C., Cortiñas, V. P., Tejera, M. I. y Del Rey, S. (2011, 7-9 de julio). James Heckman, el sesgo de selección muestral. VI Congreso Internacional de Historia de la Estadística y de la Probabilidad. Valencia España. http://www.ahepe.es/VICongreso/descargas/Cristina_Sanchez_Figueroa.pdf 
Secretaría Permanente de la Secretaría Económica Latinoamericana y del Caribe, SELA (2010). Desarrollando Mujeres Empresarias: La Necesidad de Replantear Políticas y Programas de Género en el Desarrollo de PYMES. SELA. http://iberpyme.sela.org/aDocs/Desarrollando_Mujeres_Empresarias_PYMES.pdf

Sharma, P. y Varma, S. K. (2008). Women empowerment through entrepreneurial activities of Self Help Groups. Indian Research Journal of extension education, 8(1), 46-51. https://pdfs.semanticscholar. org/88be/a1da0422ab5d1c21523a65da315f53dfb490.pdf

Sharma, Y. (2013). Women entrepreneur in India. IOSR Journal of Business and Management, 15(3), 9-14. https://pdfs.semanticscholar.org/a839 /9756d4faf59373852a9b905b1159947a93e3.pdf

Sheehy, G. (1986). Las crisis de la edad adulta. Editorial Grijalbo.

Torres, O. y Pau, B. (2011). “Techo de cristal” y suelo pegajoso. La situación de la mujer en los sistemas alemán y español de ciencia y tecnología. CTS: Revista iberoamericana de ciencia, tecnología y sociedad, 6(18), 35-59. https://dialnet.unirioja.es/servlet/articulo?codigo=3716827

Veloz, A. (2017). El sentido común sobre el género: la institucionalización del género y los sentidos del trabajo y la familia para las trabajadoras de maquiladoras en Tijuana. La ventana. Revista de estudios de género, 5(45), 120-156. https://doi.org/10.32870/lv.v5i45.5026

Willms, J. D. (2006). Learning divides. Ten policy questions about the performance and equity of schools and schooling systems. UNESCO Institute for Statistics. 